\title{
Multiple sulfatase deficiency with neonatal manifestation
}

\author{
Livia Garavelli ${ }^{1 *}$, Lucia Santoro ${ }^{2}$, Alexandra lori ${ }^{1,3}$, Giancarlo Gargano ${ }^{4}$, Silvia Braibanti ${ }^{4}$, Simona Pedori ${ }^{4}$, Nives Melli ${ }^{4}$, \\ Daniele Frattini ${ }^{5}$, Lucia Zampini², Tiziana Galeazzi ${ }^{2}$, Lucia Padella², Stefano Pepe ${ }^{6}$, Anita Wischmeijer ${ }^{1,7}$, Simonetta Rosato ${ }^{1}$, \\ Ivan Ivanovski ${ }^{1}$, Lorenzo lughetti ${ }^{3}$, Chiara Gelmini ${ }^{1}$, Sergio Bernasconi ${ }^{8}$, Andrea Superti-Furga ${ }^{9}$, Andrea Ballabio ${ }^{6,10,11,12}$ \\ and Orazio Gabrielli ${ }^{2}$
}

\begin{abstract}
Multiple Sulfatase Deficiency (MSD; OMIM 272200) is a rare autosomal recessive inborn error of metabolism caused by mutations in the sulfatase modifying factor 1 gene, encoding the formylglycine-generating enzyme (FGE), and resulting in tissue accumulation of sulfatides, sulphated glycosaminoglycans, sphingolipids and steroid sulfates. Less than 50 cases have been published so far. We report a new case of MSD presenting in the newborn period with hypotonia, apnoea, cyanosis and rolling eyes, hepato-splenomegaly and deafness. This patient was compound heterozygous for two so far undescribed SUMF1 mutations (c.191C > A; p.S64X and c.818A > G; p.D273G).
\end{abstract}

Keywords: Multiple sulfatase deficiency, MSD, SUMF1 gene

\section{Introduction}

Multiple Sulfatase Deficiency is a rare autosomal recessive inborn error of metabolism characterized by the defective activity of all known sulfatases [1]. It is caused by mutations in the sulfatases- modifying factor 1 gene encoding the FGE. Sulfatases are a family of enzymes that catalyze the hydrolysis of ester sulfates, including glycosaminoglycans, sulfolipids and steroid sulfates. These proteins share high amino acid sequence homology. However they show different sub-cellular localization and substrate specificity [1,2]. The SUMF1 gene encodes for a sulfatasemodifyng factor, which converts a highly conserved cysteine within the sulfatase catalytic domain into $\mathrm{C} \alpha$ - formylglycine [3]. It has been shown that this post-translational modification is both an essential and limiting factor for the enzymatic activity of sulfatases [4]. The SUMF1 gene is located on chromosome 3p26. It spans $105 \mathrm{~kb}$ and the coding sequence is distributed over 9 exons. The cDNA for human FGE is predicted to encode a protein of 374 residues. The protein contains a cleavable signal sequence of 33 residues, which indicates translocation of FGE

\footnotetext{
* Correspondence: garavelli.livia@asmn.re.it

${ }^{1}$ Clinical Genetics Unit, Obstetric and Pediatric Department, Istituto di Ricovero e Cura a Carattere Scientifico, Arcispedale Santa Maria Nuova, Reggio Emilia, Italy

Full list of author information is available at the end of the article
}

into the endoplasmatic reticulum, and contains a single $\mathrm{N}$-glycosylation site at Asn-141 [5]. The middle part of FGE (residues 179-308 in human FGE) is represented by a tryptophan-rich subdomain. The $\mathrm{C}$-terminal subdomain (residues 327-366 in human FGE) is the most highly conserved sequence within the FGE family.

FGE post translationally activates all newly synthesized sulfatase by generating the catalytic residue formylglycine. Impaired FGE function leads to a reduction in sulfatase activities. It has been proved that variability of the clinical phenotype depends on both residual FGE activity as well as protein stability [6]. MSD is characterized by features of mucoplysaccharidosis and metachromatic leukodystrophy including neurologic deterioration and development delay, gargoyle-like features, visceromegaly, heart involvement, ichthyosis, hydrocephaly, cloudy corneal and skeletal anomalies [7]. According to the age of onset, very neonatal, late infantile (severe or LIS and mild or LIM) and rare juvenile (mild) disease subtypes can be distinguished [8].

We described a clinical case of a girl with a neonatal form of MSD, presenting typical findings of the disease. She was compound heterozygous for two so far undescribed SUMF1 mutations (c.191C > A; p.S64X and c.818A > G; p.D273G). 


\section{Clinical report}

The girl is the first child of healthy, non-consanguineous parents. She was born by vaginal delivery at week 40 with birth weight of $2,815 \mathrm{~g}$, length $46 \mathrm{~cm}$, and head circumference $32 \mathrm{~cm}$. APGAR scores were 9/10. At 20 hours of life she was transferred to our Neonatal Intensive Unit because of the appearance of rolling eyes followed by episodes of apnoea with cyanosis and hypotonia, for which a treatment with phenobarbital was installed. At that time, physical examination showed coarse facial features, higharched, thick eyebrows, bulbous nasal tip, micrognathia, posteriorly rotated ears with attached lobes, flared thorax, inverted nipples, hepatosplenomegaly, broad thumbs, mild ichthyosis and muscular hypotonia. (Figure 1A, B, C, D). At 11 days of life she presented two episodes of obstructive respiratory arrest after stridor, treated with ventilation. The otolaryngologist's evaluation revealed the presence of a cystic formation of the vallecula, which was removed surgically with resolution of the episodes of apnoea.

Because of the coarse facial features, hypertrichosis, and hepatosplenomegaly, a lysosomal storage disorder

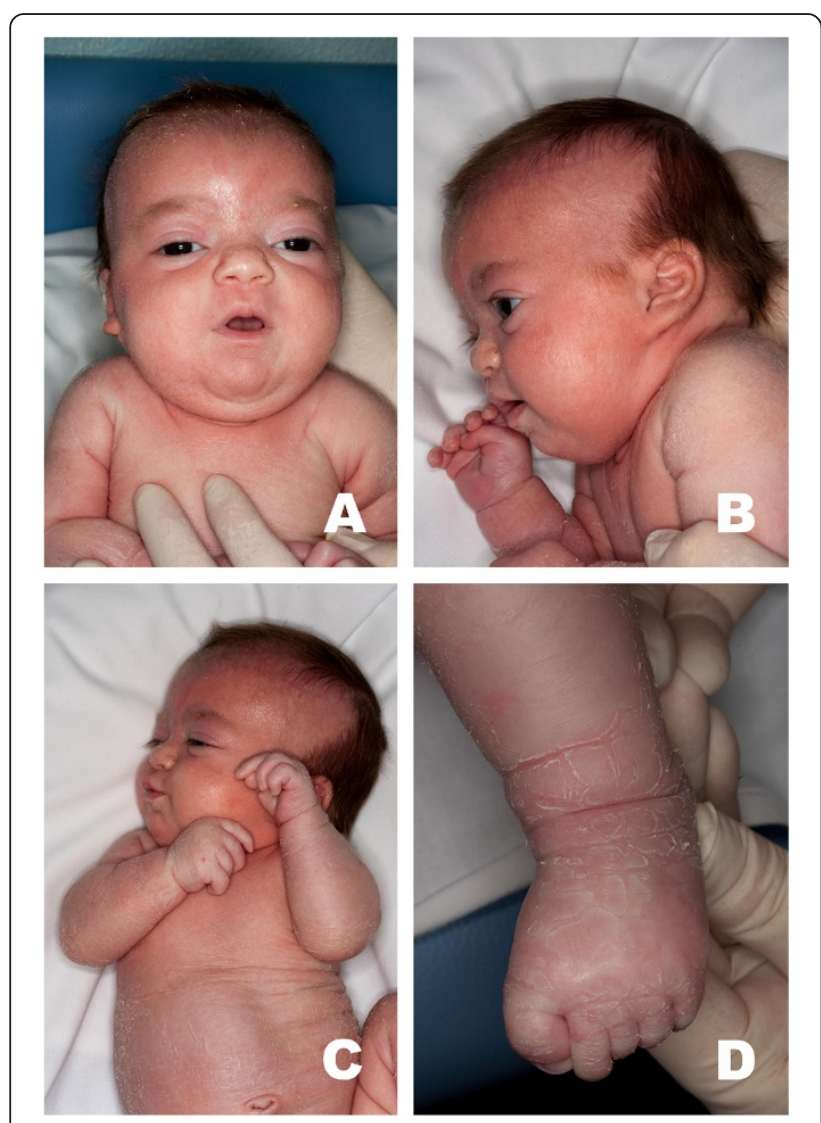

Figure 1 Phenotype. A: coarse face, high-arched, thick eyebrows, bulbous nasal tip and mild ichthyosis. B: micrognathia, posteriorly rotated ears with attached lobes. C: abdominal ichthyosis. D: ichthyosis of the lower limb and feet. was considered. There was an increased urinary excretion of glycosaminoglycans (1101 $\mu \mathrm{g} / \mathrm{mg}$ creatinine; normal range, 30-200), with chondroitin sulfate $(+++)$, dermatan sulfate $(+++)$ and heparan sulfate $(++)$ on electrophoresis. Enzyme activitiy testing in leucocytes showed deficiency of arylsulfatase A (0.0 nM/mg/h; n.v. 40-270), arylsulfatase $\mathrm{B}(0.0 \mathrm{nM} / \mathrm{mg} / \mathrm{h} ; \mathrm{n} . v$. 70-126), iduronate sulfatase (0.9 nM/mg/4 h; n.v. 51-123) and galactose 6 sulfatase (0.6 nM/mg/17 h; n.v.20-27). This pattern was diagnostic of Multiple sulfatase deficiency (MSD). Molecular analysis of the gene SUMF1 revealed two so far undescribed heterozygous mutations: c.191C > A s. S64X in exon 1 and c.818A > G p.D273G in exon 6. The first heterozygous mutation was also demonstrated in the girl's mother, while the second one was identified in the girl's father.

The karyotype was normal, 46,XX. The echocardiography and EEG were normal; abdominal ultrasound showed hepatomegaly; brain magnetic resonance imaging (MRI) showed dilated lateral ventricles with an enlarged cisterna magna, demyelination of the white matter, partial corpus callosum hypoplasia with hypothrophy of the splenium and dismorphism of the hippocampum. Skeletal radiography revealed a pattern of dysostosis multiplex with hypoplasia of L2, lumbar kyphosis and platyspondyly of the cervical vertebral bodies; broad ribs; broad and hypoplastic first metacarpals, pointing of the second and third metacarpals, irregularity of the distal radial and ulnar metaphysis (Figure 2A, B, C, D). The ABR examination showed mild hypoacusia.

The child presented global developmental delay: could hold head up only at the age of 10 months, at the age of 12 months she was still hypotonic, with limited and jerky movements and it was impossible to meet her gaze. She developed dorso-lumbar kyphosis and owing to L2 hypoplasia she had to wear a orthopaedic corset.

She was seen again in our Clinical Genetics Unit at 24 months of age. Her head circumference was $45 \mathrm{~cm}$ (50th centile), length $66 \mathrm{~cm}$ ( $<3 \mathrm{rd}$ centile), and weight $6.406 \mathrm{Kg}(<<3$ rd centile). Her psychomotor development was severely delayed and she uttered no words. At the age of 24 months she could not sit up unaided.

\section{Discussion}

Multiple sulfatase deficiency is a rare autosomal recessive inborn error of metabolism affecting post-translational activation of sulfatases by the formylglycine generating enzyme (FGE). Due to mutations in the encoding SUMF1 gene, FGE's catalytic capacity is impaired, resulting in reduced cellular sulfatase activities [9]. More than 30 different SUMF1 mutations are known, most of them missense mutations that affect stability and residual molecular activity of mutant FGE, which both determine MSD disease severity [10]. A variable residual activity of the different sulfatases has been described $[1,11]$. 

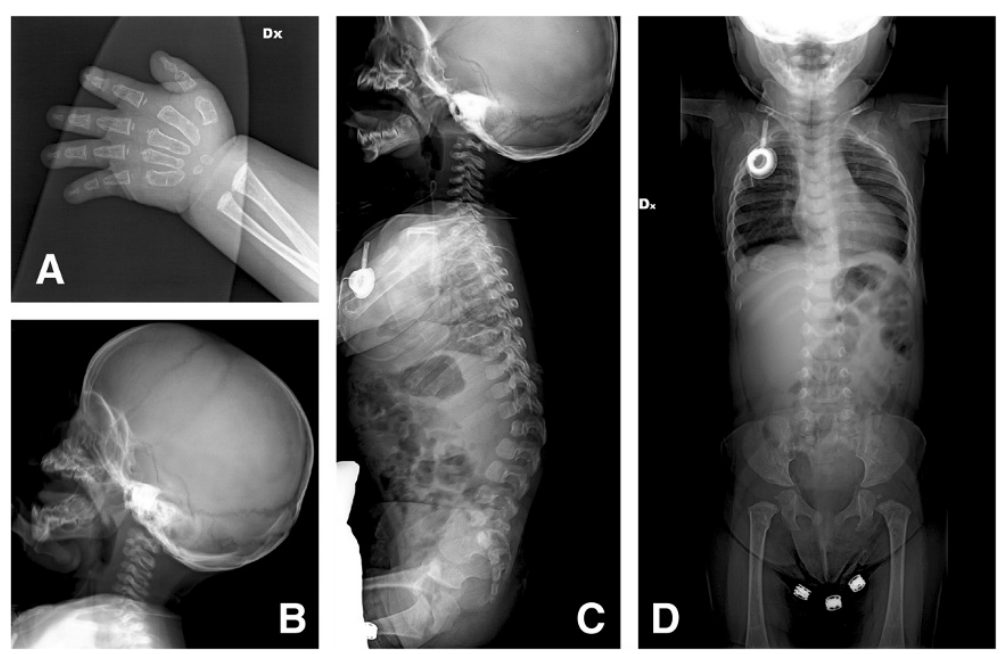

Figure 2 X-Rays. A: broad and hypoplastic first metacarpals, pointing of the second and third metacarpals, irregularity of the distal radial and ulnar metaphysis. B: platyspondily of the cervical vertebral bodies. C: hypoplastic vertebral body of L2, lumbar kyphosis. D: broad ribs.

Few studies were able to show a correlation of both residual activity and stability of FGE variants with the clinical presentation of selected MSD patients. Patients with drastic impairments of both FGE stability and residual enzyme activity displayed the most severe clinical phenotype whereas the mildest phenotype was associated with the highest residual FGE activity among the studied variants.

It is now possible to establish a genotype/phenotype correlation for MSD and to roughly predict the clinical course for patients with the studied SUMF1 mutations. Missense mutations affecting crucial functional or structural residues in FGE, as well as nonsense ones, will cause severe forms of the disease, whereas missense mutations not fully abrogating a functional conformation of the FGE protein will lead to attenuated forms [6].

Different types of MSD can be distinguished according to the age of onset: neonatal, late infantile $(0$ to 2 years, severe or LIS and mild or LIM), and rare juvenile (2 to 4 years, mild) [8].

The clinical picture of MSD combines symptoms of the different sulfatase deficiencies. Patients show neurological deterioration and a neurodegenerative course of disease similar to metachromatic leukodystrophy. Development delay, dysmorphism and organomegaly are present as found in various mucopolysaccharidoses [6]. Skeletal abnormalities remind one of Chondrodysplasia punctata type 1 and skin changes of X-linked ichthyosis [1].

The onset of symptoms in early infancy is associated with poor chances of survival; conversely, when features of the disease appear in late infancy, patients survive longer.

In general it is rare to carry out a diagnosis of storage disease in newborns apart from I-cell disease also known as mucolipidosis II, which is a condition with a severe clinical course, typical radiological aspects and fibroblast inclusions. Other symptoms include hyperplastic gums, thoracic deformities and congenital hip dislocation which are evident in newborn age. In the other storage diseases the symptoms are not so evident in the newborn age.

The diagnosis of MSD may not be simple due to the rarity of the condition: in our case the features which led us to suspect the diagnosis were the MPS-like phenotype, the radiological signs compatible with "dysostosis multiplex" and especially the cutaneous features. The child had no signs of chondrodysplasia punctata, in particular no epiphyseal stippling.

Indeed the presence of ichthyosis can be a very useful indicator in identifying the condition and can be the best marker of the disease in newborns.

Our patient had typical features of neonatal MSD including neonatal hypotonia, coarse face, mild deafness, visceromegaly, hypoplastic vertebral bodies and delayed psychomotor development.

It was demonstrated that the phenotypic outcome depends on both residual FGE activity as well as protein stability [6]. Another neonatal case was described which showed all the clinical symptoms of the condition and a quick worsening course of the disease: he presented two nonsense mutations leading to almost fully abrogated FGE activity, highly unstable FGE protein and nearly undetectable sulfatase activity [6]. Our case presents a missense mutation along with a nonsense mutation.

In fact we have identified two so far undescribed SUMF1 mutations (c.191C > A; p.S64X and c.818A > G; p.D273G), resulting in a severe clinical phenotype with early onset, but which allow survival beyond one year of life, in contrast with what has been reported in literature up to this point. She has a serious clinical aspect, but probably less 
severe than that described by Schlotawa and is still alive at the age of 2 years.

MSD is still an untreatable disease. Thus, potential treatment approaches, and also genetic counselling, directly depend on thorough analyses of the functional consequences of human SUMF1 mutations on the clinical and biochemical phenotype.

Understanding better the molecular mechanism at the root of this condition could aid the identification of the most appropriate therapeutic approach of MSD in the near future.

\section{Conclusion}

In conclusion, our patient illustrates well the main clinical features to suspect this condition in the newborn age, ichthyosis in particular, the wide spectrum of clinical manifestations and biochemical abnormalities of this rare disease. This case serves to highlight the importance of full and detailed evaluation of all patients with rare disorders and the need for continued updates of suggested surveillance for these diseases.

\section{Consent}

Written informed consent was obtained from the patient's guardian/parent/next of kin for the publication of this report and any accompanying images".

\section{Competing interests}

The authors declare that they have no competing interests.

\section{Authors' contributions}

$L G$ and $A$ l wrote the manuscript, $L G, L S, G G, S B, N M, D F, L Z, T G, L P, A W$, $S R, I I, L I, C G, S B, A S-F$ and $O G$ participated in the diagnostic process, AS-F evaluated the X-Rays, SP and AB carried out the molecular genetic tests. All authors read and approved the final manuscript.

\section{Acknowledgements}

The authors wish to thank the co-operating family-members for the necessary medical data and photographs for publication as well as the photographers Marco Bonazzi and Luca Valcavi. This research was funded in part by Fondazione Manodori.

\section{Author details}

${ }^{1}$ Clinical Genetics Unit, Obstetric and Pediatric Department, Istituto di Ricovero e Cura a Carattere Scientifico, Arcispedale Santa Maria Nuova, Reggio Emilia, Italy. ${ }^{2}$ Pediatrics Unit, UNIVPM, Ancona, Italy. ${ }^{3}$ Department of Medical and Surgical Sciences of Childhood and Adult, University of Modena and Reggio Emilia, Modena, Italy. ${ }^{4}$ Neonatal Intensive Care Unit, Obstetric and Pediatric Department, Istituto di Ricovero e Cura a Carattere Scientifico, Arcispedale Santa Maria Nuova, Reggio Emilia, Italy. ${ }^{5}$ Pediatric Neurology Unit, Obstetric and Pediatric Department, Istituto di Ricovero e Cura a Carattere Scientifico, Arcispedale Santa Maria Nuova, Reggio Emilia, Italy. ${ }^{6}$ Telethon Institute of Genetics and Medicine (TIGEM), Via Pietro Castellino 111, 80131 Naples, Italy. ${ }^{7}$ Department of Medical Genetics, Policlinico Sant'Orsola-Malpighi, University of Bologna, Bologna, Italy. ${ }^{8}$ Deparment of Pediatrics, University of Parma, Parma, Italy. ${ }^{9}$ Department of Pediatrics, Centre Hospitalier Universitaire Vaudois, University of Lausanne, Lausanne, Switzerland. ${ }^{10}$ Department of Molecular and Human Genetics, Baylor College of Medicine, Houston, TX 77030, USA. ${ }^{11} \mathrm{~J} a n$ and Dan Duncan Neurological Research Institute, Texas Children Hospital, Houston, TX 77030, USA.

${ }^{12}$ Medical Genetics, Department of Translational Medicine, Federico II University, Via Pansini 5, 80131 Naples, Italy.
Received: 11 August 2014 Accepted: 28 October 2014

Published online: 17 December 2014

\section{References}

1. Hopwood J, Ballabio A: Multiple sulfatases deficiency and the nature of the sulfatase family. In The metabolic and molecular bases of inherited disease. Edited by Mac Graw Hill. New York: Scriver CR; 2001:3725-3732.

2. Dhoot GK, Gustafsson MK, Ai X, Sun W, Standiford DM, Emerson CP Jr: Regulation of Wnt signaling and embryo patterning by an extracellular sulfatase. Science 2001, 293:1663-1666.

3. Schmidt B, Selmer T, Ingendoh A, Von Figura K: A novel amino acid modification in sulfatase that is defective in multiple sulfatase deficiency. Cell 1995, 82(2):271-278.

4. Cosma MP, Pepe S, Annunziata I, Newbold RF, Grompe M, Parenti G, Ballabio A: The multiple sulfatase deficiency gene encodes an essential and limiting factor for the activity of sulfatases. Cell 2003, 113(4):445-456.

5. Dierks T, Schmidt B, Borissenko LV, Peng J, Preusser A, Mariappan M, von Figura K: Multiple sulfatase deficiency is caused by mutations in the gene encoding the human $\mathrm{C}(\mathrm{alpha}$ )-Formylglycine generating enzyme. Cell 2003, 113(4):435-444.

6. Schlotawa L, Ennemann EC, Rahakrishnan K, Schmidt B, Chakrapani A, Christen H-J, Moser H, Steinmann B, Dierks T, Gartner J: SUMF1 mutations affecting stability and activity of formylglycine generating enzyme predict clinical outcome in multiple sulfatase deficiency. Eur I Hum Genet 2011, 19:253-261.

7. Cosma MP, Pepe S, Parenti G, Settembre C, Annunziata I, Wade-Martins R, Di Domenico C, Di Natale P, Mankad A, Cox B, Uziel G, Mancini GMS, Zammarchi E, Donati MA, Kleijer WJ, Filocamo M, Carrozzo R, Carella M, Ballabio A: Molecular and functional analysis of SUMF1 mutations in multiple sulfatase deficiency. Hum Mutat 2004, 23:576-581.

8. Eto Y, Gomibuchi I, Umezawa F, Tsuda T: Pathochemistry, pathogenesis and enzyme replacement in multiple sulfatase deficiency. Enzyme 1987, 38:273-279.

9. Schlotawa L, Radhakrishnan K, Baumgartner M, Schmid R, Schmidt B, Dierks T, Gartner J: Rapid degradation of an active formylglycine generating enzyme variant leads to a late infantile severe form of multiple sulfatase deficiency. Eur J Hum Genet 2013, 21(9):1020-1023.

10. Schlotawa L, Steinfeld R, von Figura K, Dierks T, Gartner J: Molecular analysis of SUMF1 mutations: stability and residual activity of mutant formylglycine-generating enzyme determine disease severity in multiple sulfatase deficiency. Hum Mutat 2008, 29:205.

11. Blanco-Aguirre ME, Kofman-Alfaro SH, Rivera-Vega MR, Medina C, Valdes-Flores $M$, Rizzo WB, Cuevas-Covarrubias SA: Unusual clinical presentation in two cases of multiple sulfatases deficiency. Pediatr Dermatol 2001, 18(5):388-392.

\section{Submit your next manuscript to BioMed Central and take full advantage of:}

- Convenient online submission

- Thorough peer review

- No space constraints or color figure charges

- Immediate publication on acceptance

- Inclusion in PubMed, CAS, Scopus and Google Scholar

- Research which is freely available for redistribution 